\section{The effect of tobacco additives on smoking initiation and maintenance}

\author{
Efeito dos aditivos do tabaco na iniciação e \\ manutenção do tabagismo
}

\section{El efecto de los aditivos del tabaco en el inicio del tabaquismo y su mantenimiento}

1 Insituto Nacional de Câncer, Rio de Janeiro, Brasil.

2 Faculdade de Ciências da Saúde, Universidade de Brasília, Brasília, Brasil.

${ }^{3}$ Masonic Cancer Center, University of Minnesota, Minneapolis, U.S.A.

4 Escola Nacional de Saúde Pública Sergio Arouca, Fundação Oswaldo Cruz, Rio de Janeiro, Brasil. 5 Department of Psychology, University of Waterloo Waterloo, Canada. 6 Ontario Institute for Cancer Research, Toronto, Canada.

7 Faculdade de Farmácia, Universidade Federal de Minas Gerais, Belo Horizonte, Brasil.

${ }^{8}$ Faculdade de Engenharia de Alimentos, Universidade Estadual de Campinas, Campinas, Brasil.

${ }^{9}$ National Institute for Public Health and the Environment Bilthoven, the Netherlands.

Correspondence F. J. R. Paumgartten Laboratório de Toxicologia Ambiental, Escola Nacional de Saúde Pública Sergio Arouca, Fundação Oswaldo Cruz.

Av. Brasil 4036, sala 913

Rio de Janeiro, $R J$

21040-361, Brasil.

paum@ensp.fiocruz.br
Smoking prevalence in Brazil has almost halved over the last two decades. Data from the Brazilian Institute of Geography and Statistics (IBGE) 1 shows that smoking rates among men and women aged 15 years and over fell from $33.1 \%$ in 1989 to $17.2 \%$ in 2008 . This fall in smoking prevalence has led to substantial improvements in public health in Brazil due to the strong as sociation between smoking and morbid conditions, such as chronic obstructive pulmonary disease (COPD), cancer (lung, larynx and other sites), cardiovascular morbidity, and other severe illnesses. Levy et al. 2 estimated that 420,000 smoking-attributable deaths were prevented up to 2010 , and as many as seven million projected premature deaths will be averted by 2050 . This public health success story is the result of the implementation of strict tobacco control policies, including increases in tobacco taxes and higher cigarette prices, smoke-free air laws, tobacco industry marketing bans, health warnings, mass media anti-smoking campaigns and smoking cessation treatment programs. It is anticipated that the implementation of an additional set of stricter policies would cause further reductions in tobacco-related morbidity and mortality. Using simulation modelling, Levy et al. 2 found that a further $39 \%$ reduction in smoking prevalence would prevent 1.3 million of the 9 million premature deaths projected up to 2050. In light of
Carlos Gil Moreira Ferreira 1

Dâmaris Silveira 2

Dorothy K. Hatsukami 3

Francisco José Roma Paumgartten 4

Geoffrey T. Fong 5,6

Maria Beatriz de Abreu Glória 7

Maria Cecilia de Figueiredo Toledo 8

Reinskje Talhout 9

this public health scenario, the Brazilian Health Surveillance Agency (ANVISA) has banned most flavourings and additives in tobacco products.

Over 600 substances, including flavourings, sugars, menthol, humectants, ammonium compounds, cocoa and licorice are currently added to tobacco products. Additives are mostly used in blended cigarettes consumed in Brazil, the US, Latin America, Europe (except in the UK) and a number of Asian countries. In China, Canada, the UK, Australia, Ireland and some Asian countries, Virginia type cigarettes which contain relatively few additives dominate the market. American blend cigarettes contain a mixture of tobaccos including Burley tobacco, while Virginia cigarettes are primarily composed of only one type of tobacco. Virginia tobacco is flue-cured (cured over a short period of time at high temperatures). The curing process inactivates hydrolytic enzymes leading to high sugar content. Burley tobacco, on the other hand, is cured at lower temperatures for long periods (air-curing) resulting in a low sugar and high nicotine content. According to the Brazilian Tobacco Industry Association (ABIFUMO), additives are used in American blend cigarettes to replace the sugar lost during the curing process of Burley tobacco and to give the product a consistent taste and aroma and create their own signature blend. Although ABIFUMO acknowledges the existence of additive-free blended cigarettes, 
it argues that these products are intended for a niche market and large scale production is not commercially viable.

Many scientists believe that the real purpose of using additives in cigarettes goes far beyond merely assuring a consistent taste and aroma and creating a signature blend: apart from increasing toxicity, adding a complex mixture of substances (additives) may increase the addictiveness and attractiveness of a tobacco product in terms of product appeal and palatability, which in turn is likely to have a strong impact on smoking prevalence.

Industry-sponsored studies addressing the effects of mixtures of commonly used additives on cigarette smoke chemistry and toxicity concluded that additives have no effect on the levels of chemical components of cigarette smoke and toxicity. Since the findings of these studies showed that the presence of additives increased the levels of total particulate matter (TPM) and several toxicants in mainstream smoke, these conclusions could only have been reached by expressing the levels of specific toxicants adjusted by TPM concentration. Most importantly, the tobacco industry did not carry out long-term studies or carcinogenicity assays on animals exposed by inhalation to specifically test single additives and mixtures and their pyrolysis products. Therefore, in the report (http://s.anvisa.gov.br/wps/ s/r/cXkP, accessed on 13/Jan/2015), the Working Group on Tobacco Additives (WG) did not accept the tobacco industry's claims that there is no evidence to show that additives increase the inherent toxicity of tobacco smoke.

Addiction leads to the continued exposure of smokers to toxicants, thereby contributing to the occurrence of tobacco-related diseases. Moreover, addiction per se is a medical condition that involves compulsive use despite the negative health consequences and desire to quit. The cigarette is a highly engineered and sophisticated nicotine delivery device. Although nicotine plays a major role in tobacco addiction, other substances may also play an important supporting role. In theory, additives can enhance the addictiveness of the product through increasing the overall bioavailability of nicotine by facilitating smoke inhalation and thus the absorption of nicotine through the lung alveoli, by decreasing nicotine clearance (for example due to the inhibition of CYP2A6), by changing the properties of tobacco smoke, and by enhancing the reinforcing properties of nicotine (for example monoamine oxidase inhibitors). Certain substances added to tobacco can cause one or more of these effects. Sugar in different forms is one of the most common and abundant additives in tobacco products. Al- though the oral ingestion of sugars is not hazardous to the health, the burning of sugar produces toxic aldehydes, one of which (acetaldehyde) has been shown to increase the addictiveness of tobacco in animal studies. The overall effect of a mixture of additives on smoking behaviour apparently depends on the relative amount of each component and the complex interaction between these substances. The addition of sugars, for instance, leads to an increase in acid content, lowers $\mathrm{pH}$ and reduces the harshness of smoke from cigarettes with a high nicotine/tar ratio. The absorption of nicotine decreases with decreasing smoke $\mathrm{pH}$, while substances that raise smoke $\mathrm{pH}$ (for example ammonium) facilitate absorption because the concentration of nicotine in its free uncharged form, which is more readily absorbed than positively charged nicotine, is greater at higher $\mathrm{pH}$. Therefore, a balance between the combinations of additives is apparently necessary to achieve the intended effect on smoking behaviour and product attractiveness.

Both extrinsic factors (for example marketing, pricing, packaging) and intrinsic factors (for example taste, smell, sensory attributes and pharmacological effects) influence the attractiveness of a tobacco product. Smoking initiation is influenced by smell, taste and aroma, smoke smoothness and harshness, and perceptions of environmental tobacco smoke. Tobacco companies may use additives to alter these characteristics and enhance product palatability and promote the misimpression that the resulting product is less harmful.

Studies have yet to provide evidence that supports the tobacco industry's allegations that additives have no influence on the appeal or attractiveness of tobacco products. A cross-country comparison between the US (American blend tobacco with additives) and Canada (Virginia tobacco with no or relatively few additives) which showed similar smoking rates in the two countries was presented by ABIFUMO as evidence that additives have no influence on product attractiveness. Given the differences between the confounding factors in these countries (including the type of tobacco used, configuration of additives contained in tobacco products and historical differences in the development of the tobacco market) the WG concluded that this type of cross-country comparison did not provide a valid measure of the influence of additives on the attractiveness of tobacco products.

The tobacco industry used a similar cross-country comparison to investigate the influence of additives on the inherent addictiveness of tobacco. Sanders et al. ${ }^{3}$ performed a meta-analysis of clinical studies addressing the 
effectiveness of nicotine replacement therapy and investigated differences in smoking cessation rates between countries where the tobacco market was dominated by flue-cured cigarettes (without additives) and countries where the predominant product was blended cigarettes (with additives). Based on the assumption that the difficulty of quitting smoking is a valid measure of tobacco addictiveness, Sanders et al. 3 concluded that additives do not increase cigarette addictiveness because no significant difference in quit rates was found between the countries. This sole source of evidence on the effect of additives on product addictiveness is insufficient since the study demonstrated a number of limitations, including the lack of consideration of country specific factors.

The tobacco industry claims that the effects of additives on the addictiveness and/or attractiveness of their products remain undemonstrated. Upon reviewing the studies provided by ABIFUMO and other studies identified through a comprehensive literature search, the WG concluded that the findings and arguments presented were insufficient to support any claim that additives do not enhance the addictiveness and/or attractiveness of tobacco products. Conversely, the notion that substances added to cigarettes enhance the sensory attributes of cigarette smoke and have pharmacological effects that encourage and stimulate smoking initiation and maintenance is not only scientifically plausible, but also supported by the findings highlighted by tobacco companies' reports. Documents released through litigation (Legacy Tobacco Documents Library at the University of California; http://www.legacy.library.ucsf.edu) reveal that tobacco companies were engaged in an extensive research effort to develop products that enhance or maintain nicotine delivery, increase addictiveness, improve taste and attenuate the harshness of tobacco smoke, thereby encouraging and stimulating smoking initiation and maintenance.

The burden of proof lies with the tobacco companies who have the responsibility to provide concrete scientific evidence produced through methodologically sound studies to demonstrate that additives do not enhance product addictiveness and/or attractiveness. Based on the best available evidence, it is fair to say that tobacco additives reduce the harshness of tobacco smoke and make cigarettes more attractive to consumers, thereby encouraging and stimulating smoking initiation and maintenance. A drastic reduction in smoking rates and progressive significant improvements in public health are therefore expected as ANVISA's decision to ban tobacco additives is enforced.

\section{Contributors}

All the authors participated in the drafting and revision of this text.
1. Instituto Brasileiro de Geografia e Estatística. Pesquisa Nacional por Amostra de Domicílios - Tabagismo - 2008. http://www.ibge.gov.br/home/ estatistica/populacao/trabalhoerendimento/ pnad2008/suplementos/tabagismo/ (accessed on 13/Oct/2014).

2. Levy D, Almeida LM, Szklo A. The Brazil SimSmoke policy simulation model: the effect of strong tobacco control policies on smoking prevalence and smoking-attributable deaths in a middle income nation. PLoS Med 2012; 9:e1001336.

3. Sanders E, Weitkunat R, Utan A, Dempsey R. Does the use of ingredients added to tobacco increase cigarette addictiveness? A detailed analysis. Inhal Toxicol 2012; 24: 227-45.

Submitted on $13 /$ Oct $/ 2014$

Approved on 06/Nov/2014 